\title{
EXTENSÃO UNIVERSTTÁRIA: UMA ANÁLISE DA EXPERIÊNCIA DO CURSO DE MEDICINA VETERINÁRIA DA PUCPR
}

\author{
Extension Services: an Analisys of the Experience of \\ Veterinarian Medicine Program at PUCPR
}

José Luiz Moreira ${ }^{1}$

Victor Meyer $\mathrm{Jr}^{2}$

\section{Resumo}

A extensão constitui-se em importante função da universidade ao estabelecer canais de interação com a comunidade. O objetivo desta pesquisa foi analisar a importância da contribuição das atividades de extensão desenvolvidas, pelo curso de Medicina Veterinária, no núcleo do Programa de Ação Comunitária e Ambiental - ProAção da Pontifícia Universidade Católica do Paraná, no município de Tijucas do Sul. O método de investigação que caracterizou esta pesquisa foi o estudo de caso, tendo como unidade de análise as atividades de extensão desenvolvidas pelos alunos do Curso de Medicina Veterinária em Tijucas do Sul. A população pesquisada foi constituída por alunos e ex-alunos que participaram das atividades de extensão, na forma de ação comunitánia. Os dados foram coletados por meio de questionário semi-estruturado enviado via correio eletrônico, a grupo de 51 alunos e 9 ex-alunos. 0 estudo demonstrou que, na percepção dos respondentes, as atividades de extensão, na forma de ação comunitária, apresentam uma significativa contribuição para a formação acadêmica dos alunos. Foi observado também o impacto positivo da experiência das atividades de extensão na prática profissional como apontam os ex-alunos que dela participaram. Os resultados obtidos indicam como principais contribuições das atividades de extensão a aplicação dos conteúdos desenvolvidos em sala de aula formal, a interdisciplinaridade e o contato com a realidade do meio rural.

Palavras-chave: Extensão; Formação acadêmica.

\section{Abstract}

The extension constitutes an important function of the university as it establishes interaction channels with the community. The objective of this study was to analyse the importance of the contribution of the extension activities developed by the Veterinary Medicine Course in the nucleus of the Environmental and Communitary Action Program (ProAção) of the "Pontíficia Universidade Católica do Paraná (PUCPR), located in Tijucas do Sul. The investigation method that characterized this work was the case-study method, having as the analyses unit the extension activities developed by the students of the Veterinary Medicine Course in Tijucas do Sul. The investigated population was constituted by students and former students who had participed of extension activities in the form of communitarian action. The data were collected by e-mail from 51 students and 9 former students through a half-structured questionnaire. The study demonstrated that, in the answerer's perception, the extension activities in the form of communitarian action represent a significant contribution to the academic formation of students. Is was also observed a positive impact of the extension activities experience on the professional performance as mentioned by formed students. The results obtained indicate as the main contribution of the extension activities the application of the studied contents developed in regular classroom activities, the interdisciplinary and the contact with the reality of the rural area life

Keywords: Extension; Academic formation.

1 Professor do Curso de Medicina Veterinária da PUCPR, Campus São José dos Pinhais. E-mail: jose.moreira@pucpr.br Pontifícia Universidade Católica do Paraná - PUCPR. Campus São José dos Pinhais, BR 376 - km 14-Costeira Caixa Postal 129CEP 83010-500, Tel. (41)- 3299-4300. São José dos Pinhais - Paraná.

2 Professor Pesquisador do Programa de Pós-Graduação em Educação da PUCPR, Campus Curitiba. E-mail: v.meyer@pucpr.br Pontifícia Universidade Católica do Paraná - PUCPR, Campus Curitiba.

Rua: Imaculada Conceição 1155 - Prado Velho, Caixa Postal 16.210 CEP 81611-970

Tel. (41) 3271-1655 - 3271-2242. Curitiba - Paraná. 


\section{Introdução}

A universidade contemporânea tem como função o ensino, a pesquisa e a extensão. Como ensino, entende-se a sua função mais praticada e divulgada, a transmissão do conhecimento acumulado ao longo da história da humanidade. Pesquisa é entendida como a produção do conhecimento, sendo que a terceira função, a extensão, é a de socializar o conhecimento, repassado ou produzido pela universidade, colocando-o a serviço da sociedade.

O modelo organizacional da universidade, apoiada nas três funções descritas, foi regulamentado a partir da constituição de 1988, em que a extensão universitária foi regulamentada como função das universidades brasileiras indissociável do ensino e da pesquisa (SOUSA, 2000).

A extensão universitária, implementada na forma de programas de ação comunitária, como o analisado neste estudo, assume particular importância no Brasil, como conseqüência das imensas desigualdades sociais presentes em nosso país.

No atual contexto da universidade brasileira, a extensão tem uma tarefa inquestionável: instrumentalizar o processo da práxis, contribuindo para superar o falso e o dicotômico conceito de teoria versus prática, articulando o ensino e a pesquisa com as demandas e as necessidades da sociedade, e comprometer e confrontar a comunidade acadêmica com a realidade (MARCHI, 1995).

Como exemplos de atividades de extensão freqüentes, podem ser citados cursos à comunidade, atividades recreativas, organização de corais com a participação da comunidade acadêmica e externa da universidade, bem como a prestação de serviços com o desenvolvimento e novas tecnologias, os programas de ação comunitária, entre outras.

A PUCPR, por meio de seus núcleos do ProAção (Programa de Ação Comunitária e Ambiental), coloca a serviço da comunidade acadêmica uma estrutura que tem como principal meta desenvolver atividades de extensão na modalidade de ação comunitária, que em decorrência da estrutura física e de apoio permanente, oferecem condições para a implementação, ações extensionistas com duas características bem marcantes, 0 caráter não pontual e um forte envolvimento do corpo discente, particularidades que tornam as atividades de extensão função universitária indisso- ciável das outras componentes da tríade organizacional, o ensino e a pesquisa, de forma pragmática.

O curso de Medicina Veterinária da Pontifícia Universidade Católica do Paraná participa do ProAção desde a sua implantação, em busca de atividades que possam contribuir para a formação acadêmica de seus egressos, pois a estrutura disponibilizada oferece condições para que atividades de extensão possam ser desenvolvidas de forma contínua, eliminando um fator limitador para atividades que envolvam o participação da comunidade, o caráter pontual.

O crescimento da procura, pela comunidade, dos serviços prestados, nas atividades desenvolvidas pelo curso de Medicina Veterinária da PUCPR, e a solicitação também crescente de participação dos alunos do curso nas práticas de extensão, todas de caráter voluntário, sugerem a existência de contribuições para a formação acadêmica dos alunos participantes, bem como uma forma prática de detectar a indissociabilidade entre as funções de ensino, pesquisa e extensão.

O objetivo deste trabalho foi de examinar as contribuições para a formação acadêmica dos alunos e ex-alunos da Pontifícia Universidade Católica do Paraná, participantes das atividades de extensão desenvolvidas pelo curso de Medicina Veterinária na unidade do ProAção de Tijucas do Sul, município distante 70 quilômetros de Curitiba. A fonte da informação foi a percepção dos alunos e ex-alunos envolvidos nas atividades descritas desde a sua implantação.

\section{Materiais e métodos}

O estudo caracteriza-se como um estudo de caso, uma vez que seu foco de análise se concentrou em uma atividade específica de extensão, desenvolvida pelo Curso de Medicina Veterinária oferecido pela Pontifícia Universidade Católica do Paraná. De acordo com Bruyne, Herman e Schauthute (1982), o estudo de caso consiste em uma análise intensiva, empreendida em uma organização e que busca reunir informações tão numerosas e tão detalhadas quanto possível, com vistas a apreender a totalidade de uma situação. Ainda segundo Toni (2000), no estudo de caso, é realizado um levantamento de vários dados da realidade que se pretende conhecer. 
Os dados deste trabalho de investigação foram obtidos por intermédio de questionários semi-estruturados, aplicados junto a alunos que atualmente participam das atividades no referido núcleo, bem como a alunos egressos do curso de Medicina Veterinária da PUCPR, que participaram das atividades de extensão no núcleo do ProAção de Tijucas do Sul.

Foram aplicados dois instrumentos de pesquisa no presente estudo, um destinado aos atuais participantes das atividades de extensão, implementadas pelo curso de Medicina Veterinária, no núcleo do ProAção de Tijucas do Sul, e um segundo que teve como foco os ex-participantes, que hoje estão inseridos no mercado de trabalho, ou realizando cursos de pós-graduação.

Foram distribuídos 51 instrumentos de pesquisa, para alunos que estão freqüentando 0 curso de graduação em Medicina Veterinária da
PUCPR, participantes das atividades de extensão, regularmente matriculados no curso de graduação. Os dados foram coletados na segunda quinzena do mês de junho de 2004, tanto para alunos, como para ex-alunos.

Foram enviados aos endereços eletrônicos dos ex-alunos participantes das atividades de extensão, localizados pelo cadastro da PUCPR e de informações de colegas do curso, 15 questionários, dos quais obtivemos efetiva resposta de 9 exemplares.

\section{Resultados e discussões}

A análise dos dados coletados apresenta uma clara contribuição, na percepção dos alunos entrevistados, das atividades de extensão na sua formação acadêmica.

\section{TABEIA 1 - Período que o aluno está freqüentando o curso de Medicina Veterinária.}

Table 1 - Student participation in Veterinary Medicine course by semesters.

\begin{tabular}{|c|c|c|}
\hline Período & Freqüência absoluta & Frequiência Relativa (\%) \\
\hline $3 .^{\circ}$ Período & 9 & $17,5 \%$ \\
\hline 4. ${ }^{\circ}$ Período & 5 & $10 \%$ \\
\hline 5. ${ }^{\circ}$ Período & 15 & $29 \%$ \\
\hline 6. ${ }^{\circ}$ Período & 7 & $14 \%$ \\
\hline 7. ${ }^{\circ}$ Período & 9 & $17,5 \%$ \\
\hline 8. ${ }^{\circ}$ Período & 4 & $8 \%$ \\
\hline 9. ${ }^{\circ}$ Período & 2 & $4 \%$ \\
\hline Total & 51 & $100 \%$ \\
\hline
\end{tabular}

Fonte: Entrevista semi-estruturada.

Ao se examinar, na tabela 1 , a fase do curso de graduação a que pertencem os alunos que participam das atividades de extensão, verifica-se que 15 (quinze) alunos, ou seja, 29\% dos respondentes pertence ao quinto período do curso de graduação, de um total de 51 alunos respondentes. Esses alunos iniciaram a participação nas atividades quando cursavam o terceiro período e permaneceram participando dessa atividade.

Quanto aos demais participantes, verificou-se uma diversidade de fases do curso de graduação. Isto se deve ao fato de que os alunos são incentivados pelos coordenadores das atividades de extensão a participar desde os primeiros semestres do Curso. O objetivo é proporcionar uma convivência entre alunos das diferentes fases do Curso de Medicina Veterinária, incentivando a troca de informações entre eles, e a realização de atividades extensionistas, em equipe. Este fato foi valorizado principalmente pelos ex-alunos já atuando profissionalmente no mercado de trabalho. 


\section{TABELA 2 - Fator motivador de participação dos alunos nas atividades.}

Table 2 - Motivator factor of student participation in the activities.

\begin{tabular}{l|c|c}
\hline \multicolumn{1}{c|}{ Fator } & Freqüência absoluta & Freqüência Relativa (\%) \\
\hline - Necessidade de realizar atividades práticas & 10 & $20 \%$ \\
- Incentivo por parte de colegas do curso. & 1 & $2 \%$ \\
- Conhecimento da realidade da rotina profissional. & 28 & $55 \%$ \\
- Outra & 12 & $23 \%$ \\
\hline Total & 51 & $100 \%$ \\
\hline
\end{tabular}

Fonte: Entrevista semi-estruturada.

Conforme se observa dos dados apresentados na Tabela 2, a maioria (55\%) dos alunos entrevistados apontou "conhecer a rotina profissional" como o principal motivo para participar das atividades de extensão. Para $23 \%$ dos respondentes, o principal motivo para participação foi a "necessidade de realizar atividades práticas" e "conhecer a realidade da rotina profissional". Nota-se aqui uma clara preocupação dos alunos respondentes em familiarizar-se com as rotinas da futura profissão. A prática sem teoria é um adestramento tolo, apropriado em pouquíssimos casos. E a teoria sem prática é considerada viável para uma ínfima minoria que viceja na abstração pura. A maioria dos alunos precisa de prática para entender a teoria (CASTRO, 2003).

\section{TABELA 3 - Aplicação dos conteúdos de sala de aula nas atividades.}

Table 3 - Application of the lectures in the activities.

\begin{tabular}{cc|c|c}
\hline & & Freqüência absoluta & Freqüência Relativa (\%) \\
\hline- & Sim & 51 & $100 \%$ \\
$1-$ & Não & 0 & $0 \%$ \\
\hline Total & & 51 & $100 \%$ \\
\hline Fonte: Entrevista semi-estruturada. & & \\
\hline
\end{tabular}

Quando perguntados a respeito da aplicação dos conteúdos desenvolvidos em sala de aula nas atividades de extensão, todos os respondentes foram unânimes em afirmar que as atividades de extensão desenvolvidas em Tijucas do Sul proporcionam aplicação dos conteúdos incorporados em sala de aula. Destaque-se que o curso de Medicina Veterinária tem sempre desenvolvida uma política de apoiar, de forma irrestrita, as atividades extensionistas, por acreditar na efetiva integração entre ensino e extensão, contribuindo para a formação acadêmica dos alunos e enriquecimento das atividades acadêmicas do corpo docente. Isto reforça a proposta de Morin (2003) de que a educa- ção do século XXI deve ser contextualizada e estar inserida na realidade.

Ao constatar a aplicação dos conteúdos, é de se esperar que este aprendizado se torne profundo e o contato com a comunidade e a realidade profissional um componente do "curnículo oculto" que as atividades de extensão proporcionam a seus participantes.

O aluno, ao relacionar as teorias apreendidas em sala de aula com sua aplicação prática, tem a oportunidade de eliminar a velha indagação, "para quê tenho que estudar" isto, ou ainda "para que serve este conteúdo", beneficiando-se academicamente da integração ensino e extensão. 
TABELA 4 - Resultado da interdisciplinaridade percebida nas atividades de extensão.

Table 4 - Results of the course interaction evaluated in the extension activities.

\begin{tabular}{l|c|c}
\hline & $\begin{array}{c}\text { Freqüência } \\
\text { absoluta }\end{array}$ & $\begin{array}{c}\text { Freqüência } \\
\text { Relativa (\%) }\end{array}$ \\
\hline- Sim & 51 & $100 \%$ \\
- Não & 0 & $0 \%$ \\
\hline Total & 51 & $100 \%$ \\
\hline Fonte: Entrevista semi-estruturada.
\end{tabular}

Fonte: Entrevista semi-estruturada.

Os dados contidos na Tabela 4 demonstram que a totalidade dos respondentes entende haver interdisciplinaridade nas atividades de extensão. A divisão de um curso em programas de aprendizagem, a partir do novo projeto pedagógico implementado na PUCPR, a partir do ano de 2000, requer a ordenação de conteúdos. Esta seqüência de conteúdos tem sido muito questionada pela área pedagógica das instituições de ensino, classificando-a como o ensino fragmentado e descontextualizado.

Observa-se, dos dados da Tabela 4, que $100 \%$ dos respondentes destacam que as atividades de extensão contribuem para a interdisciplina- ridade ao facilitar a contextualização de conteúdos teóricos e faz com que o aluno consiga visualizar o envolvimento e a interdependência entre os conteúdos dos programas de aprendizagem integrantes do currículo do Curso de Medicina Veterinária da PUCPR.

Ao se analisar a percepção dos ex-alunos que participaram das atividades de extensão de Tijucas do Sul, observa-se não maior discrepância em relação às contribuições dessas atividades para a formação acadêmica. Uma das dimensões importantes é que este grupo de respondentes identifica contribuição efetiva das atividades extensionistas de Tijucas do Sul em sua atividade profissional.

\section{TABELA 5 - Função desempenhada pelos alunos.}

Table 5 - Function role played by the students.

\begin{tabular}{|c|c|c|}
\hline Função & $\begin{array}{c}\text { Frequiência } \\
\text { absoluta }\end{array}$ & $\begin{array}{c}\text { Frequiência } \\
\text { Relativa (\%) }\end{array}$ \\
\hline $\begin{array}{l}\text { - Residente em clínica e cirurgia de grandes animais } \\
\text { - Residente em Clínica, Cirurgia e Reprodução de } \\
\text { Ruminantes }\end{array}$ & $\begin{array}{l}02 \\
01\end{array}$ & $\begin{array}{c}22,5 \% \\
11 \%\end{array}$ \\
\hline - Médico Veterinário, de Clínica de Animais de Companhia & 02 & $22,5 \%$ \\
\hline - Anestesiologista Veterinário & 01 & $11 \%$ \\
\hline - Coordenadora de trademarketing - Bayer Saúde Animal & 01 & $11 \%$ \\
\hline - Medicina Veterinária Autônoma & 01 & $11 \%$ \\
\hline - Mestrando em Medicina Veterinária & 01 & $11 \%$ \\
\hline Total & 09 & $100 \%$ \\
\hline
\end{tabular}

Fonte: Entrevista semi-estruturada.

Ao se examinar os dados da Tabela 5, verifica-se que os ex-alunos desenvolvem atividades diversificadas dentro do campo de atuação da Medicina Veterinária, como a área de animais de companhia, animais de produção, empresa de produtos animais. Um dos respondentes freqüenta programa de pós-graduação stricto sensu em outra instituição de ensino. 


\section{TABELA 6 - Resultados concretos da participação dos estudantes no programa de extensão desenvolvido em Tijucas do Sul.}

Table 6 - Concrete results of the students participation in the extension program developed in Tijucas do Sul.

\begin{tabular}{|c|c|c|}
\hline Resultados & $\begin{array}{l}\text { Freqüência } \\
\text { absoluta }\end{array}$ & $\begin{array}{l}\text { Freqüência } \\
\text { Relativa }(\%)\end{array}$ \\
\hline $\begin{array}{l}\text { P ossibilitaram a constatação real dos conteúdos } \\
\text { teóricos, discutidos ao longo do curso }\end{array}$ & 01 & $11 \%$ \\
\hline ossibilitaram adquirir práticas de rotina profissional & 01 & $11 \%$ \\
\hline $\begin{array}{l}\text { P ossibilitaram vivenciar as práticas de rotina } \\
\text { profissional, inseridas na realidade do meio rural. } \\
\text { O utra }\end{array}$ & $\begin{array}{l}06 \\
01\end{array}$ & $\begin{array}{l}67 \% \\
11 \%\end{array}$ \\
\hline Total & 09 & $100 \%$ \\
\hline
\end{tabular}

Fonte: Entrevista semi-estruturada.

Observa-se, na Tabela 6, que 67\% dos exalunos pesquisados entendem que "vivenciar práticas da rotina profissional do Médico Veterinário, inseridas na realidade do meio rural", como o principal benefício da participação nas atividades de extensão de Tijucas do Sul. Isto reforça a relevância da atividade extensionista desenvolvida e sua pertinência quanto à realidade, vindo ao encontro do que enfatiza Morin (2003), já destacado neste trabalho.

Um dos respondentes, item "outro", destacou "a segurança que hoje possuo para receitar e clinicar adquiri principalmente nas atividades de Tijucas do Sul". Esta citação ressalta, uma vez mais, a real contribuição das atividades de extensão para a formação acadêmica e também para a prática profissional.

TABELA 7 - Benefícios do contato com a comunidade para a formação acadêmica.

Table 7 - Benefits of the contact with the community for the academic formation.

\begin{tabular}{|c|c|c|}
\hline Benefícios & $\begin{array}{l}\text { Frequiência } \\
\text { absoluta }\end{array}$ & $\begin{array}{l}\text { Freqüência } \\
\text { Relativa }(\%)\end{array}$ \\
\hline $\begin{array}{l}\text { - C o n hecer procedimentos populares de Medicina } \\
\text { Veterinária. }\end{array}$ & 00 & $0 \%$ \\
\hline $\begin{array}{l}\text { - A d q uirir práticas de retina profissional do Médico } \\
\text { Veterinário, nas condições do meio rural. }\end{array}$ & 01 & $11 \%$ \\
\hline $\begin{array}{l}\text { - E xercer rotina profissional, dentro das limitações } \\
\text { impostas pela realidade sociocultural do meio rural. } \\
\text { - Outra }\end{array}$ & $\begin{array}{l}07 \\
01\end{array}$ & $\begin{array}{l}78 \% \\
11 \%\end{array}$ \\
\hline Total & 09 & $100 \%$ \\
\hline
\end{tabular}

Fonte: Entrevista semi-estruturada. 
Esta tabela traduz a contribuição das atividades de extensão para a formação acadêmica, tendo como foco principal a possibilidade de implementar atividades inerentes à profissão do Médico Veterinário, inseridas nas limitações que a realidade impõem, sejam de natureza social, cultural ou econômica. Ao ser inserido no mercado de trabalho, o aluno já possui uma visão da sua rotina profissional dentro da realidade e, principalmente, das limitações que esta impõe.

Ao serem perguntados se as atividades de extensão desenvolvidas em Tijucas do Sul devem ser recomendadas aos estudantes do Curso de Medicina Veterinária da PUCPR, a totalidade dos ex-alunos que responderam à entrevista foi unânime em apontá-las como positivas. Isto reflete que as contribuições acadêmicas e profissionais decorrentes das atividades de extensão são consideradas importantes pelos ex-alunos que agora atuam profissionalmente no mercado de trabalho.

\section{Considerações finais}

Observa-se, nos dados coletados, que as atividades de extensão desenvolvidas em Tijucas do Sul contribuem efetivamente para a formação acadêmica dos alunos participantes.

Sob o ponto de vista técnico, a experiência extensionista permite aos alunos conhecer a realidade da prática profissional, durante o curso de graduação, tornando e ensino contextualizado.

Do ponto de vista social, a experiência extensionista de Tijucas do Sul contribui significativamente para a dos alunos, pelo contato com a realidade da comunidade carente, nela atuando e interagindo.

Com base na percepção de ex-alunos, observam-se também reflexos positivos das atividades de extensão na prática profissional, reforçando a relevância da extensão, como canal de integração da universidade com o setor produtivo e com a sociedade.

\section{Referências}

BRUYNE, P.; HERMAN, J.; SCHOUTHEETE, M. Dinâmica de Pesquisa em Ciências Sociais. 2. ed. Rio de Janeiro, RJ: Francisco Alves, 1982.

CASTRO, Cláudio de Moura. Os dinossauros e as gazelas do ensino superior. In: MEYER JR, Victor; MURPHY, Patrick. Dinossauros, gazelas e tigres: novas abordagens da administração universitária: um diálogo Brasil e EUA. Florianópolis: Insular, 2003.

MARCHI, Euclides. Ensino e extensão: uma indissociabilidade natural. Perfil da Extensão Universitária: cadernos de extensão, Curitiba, v. 1, n.1, p. 40-43, out. 1995.

MORIN, Edgar. Os sete saberes necessários à educação do futuro. São Paulo, SP: Cortez, 2000.

SOUSA, Ana Luiza Lima. A história da extensão universitária. Campinas: Alínea, 2000.

TONI, Adriana de. Promoção de ações de cidadania por intermédio de programas de extensão universitária: um estudo de caso na UNOESC Chapecó. Dissertação (Mestrado) - UFSC, Florianópolis, 2000.
Recebido: 03/02/2004

Aprovado: 30/06/2004 